\title{
Primary Cilia Regulate Proliferation of Amplifying Progenitors in Adult Hippocampus: Implications for Learning and Memory
}

\author{
Alejandro Amador-Arjona, ${ }^{1}$ Jimmy Elliott, ${ }^{1,3}$ Amber Miller, ${ }^{1}$ Ashley Ginbey, ${ }^{1}$ Gregory J. Pazour, ${ }^{4}$ Grigori Enikolopov, ${ }^{5}$ \\ Amanda J. Roberts, ${ }^{2}$ and Alexey V. Terskikh ${ }^{1}$ \\ ${ }^{1}$ Sanford-Burnham Medical Research Institute, and ${ }^{2}$ Molecular and Integrative Neurosciences Department, The Scripps Research Institute, La Jolla, \\ California 92037, ${ }^{3}$ Genomic Institute of the Novartis Research Foundation, San Diego, California 92121, ${ }^{4}$ University of Massachusetts Medical School, \\ Worcester, Massachusetts 01605, and ${ }^{5}$ Cold Spring Harbor Laboratory, Cold Spring Harbor, New York 11724
}

Integration of new neurons into the adult hippocampus has been linked to specific types of learning. Primary cilia were found to be required for the formation of adult neural stem cells (NSCs) in the hippocampal dentate gyrus during development. However, the requirement of cilia in maintenance of adult NSCs is unknown. We developed a genetic mouse model in which fetal/perinatal brain development is unaffected, but adult hippocampal neurogenesis is constantly reduced by conditional ablation of primary cilia in adult GFAP ${ }^{+}$neural stem/progenitor cells. We found that this approach specifically reduces the number of hippocampal amplifying progenitors (also called type 2a cells) without affecting the number of radial NSCs (or type 1 cells). Constant reduction of adult hippocampal neurogenesis produced a delay rather than a permanent deficiency in spatial learning without affecting the retention of long-term memories. Decreased neurogenesis also altered spatial novelty recognition and hippocampus-independent cue conditioning. Here, we propose that adult hippocampal newborn neurons increase the efficiency of generating the new representations of spatial memories and that reduction of adult hippocampal neurogenesis may be biased toward cue-based strategies. This novel mouse model provides evidences that cognitive deficits associated with ciliary defects (ciliopathies) might be, in part, mediated by the deficiency of primary cilia in adult hippocampal stem/progenitor cells.

\section{Introduction}

Primary cilia are specialized cellular organelles functionally implicated in adult neurogenesis within hippocampal dentate gyrus (DG) (Breunig et al., 2008; Han et al., 2008). Interestingly, primary cilia are required for sonic hedgehog (Shh) signaling (Corbit et al., 2005; Rohatgi et al., 2007), a mitogenic pathway that controls the proliferation of neural progenitors (Lai et al., 2003; Palma et al., 2005). Although previous studies have described that primary cilia and Shh are essential for the formation of adult hippocampal neural stem cells (NSCs) (Machold et al., 2003; Breunig et al., 2008), their role in the maintenance of adult stem/ progenitor cell populations is still unknown.

In the DG, adult stem/progenitor cells are located in the subgranular zone (SGZ) (Seri et al., 2001; van Praag et al., 2002). Phenotypically, they are generally classified into two different cell

Received Feb. 28, 2011; revised April 5, 2011; accepted April 7, 2011.

Author contributions: A.A.-A., A.J.R., and A.V.T. designed research;A.A.-A., J.E., A.M., A.G., and A.J.R. performed research; G.J.P. and G.E. contributed unpublished reagents/analytic tools; A.A.-A., J.E., G.E., A.J.R., and A.V.T. analyzed data; A.A.-A., A.J.R., and A.V.T. wrote the paper.

This work has been supported by transient research support from the Sanford-Burnham Medical Research Institute (A.V.T.) and NIH Blueprint Core Grant NS057096 (principal investigator, S. A. Lipton). We thank M. V. Sofroniew (University of California, Los Angeles, Los Angeles, CA) for mGFAP-Cre mice. We also thankF. H. Gage (The Salk Institute, La Jolla, (A) for discussions and sharing findings prior to submission.

Correspondence should be addressed to either Alejandro Amador-Arjona or Alexey V. Terskikh, Department of Stem Cells and Regeneration, Sanford-Burnham Medical Research Institute, 10901 North Torrey Pines Road, La Jolla, California, CA 92037.E-mail: aamador@sanfordburnham.org or terskikh@sanfordburnham.org.

DOI:10.1523/JNEUROSCI.1062-11.2011

Copyright $\odot 2011$ the authors $\quad 0270-6474 / 11 / 319933-12 \$ 15.00 / 0$ populations: (1) radial NSCs (or type 1 cells), with a radial glial fibrillary acidic protein (GFAP) process, that cross the granule cell layer (GCL), express NESTIN and the SRY-related HMG (high mobility group) box transcription factor SOX2, and rarely enter into cell cycle; and (2) amplifying progenitors (or type $2 \mathrm{a}$ cells), which express NESTIN and SOX2 but do not have the radial GFAP process, and enter into cell cycle more often (Garcia et al., 2004; Seri et al., 2004; Steiner et al., 2006). The type 2a cells were propose to be derived from type 1 cells (Garcia et al., 2004; Seri et al., 2004); however, other nonradial SOX2 ${ }^{+}$quiescent stem cells may exist (Suh et al., 2007).

In the hippocampal DG, adult stem/progenitor cells generate new neurons throughout life that integrate into preexisting circuits (van Praag et al., 2002). Among the diverse approaches to study the contribution of adult newborn hippocampal neurons in learning and memory, genetic targeting of neural precursors (Dupret et al., 2008; Imayoshi et al., 2008; Zhang et al., 2008) has emerged as an alternative method to reduce the multiple adverse effects of previous models, such as low-dose brain irradiation. However, genetic manipulations to reduce adult neurogenesis have also yielded contradictory results (Saxe et al., 2006; FarioliVecchioli et al., 2008; Deng et al., 2009), in part due to ablation of neural precursors or newborn DG neurons at different immature stages.

Here, we developed a genetic mouse model of continuous reduction in adult hippocampal neurogenesis. Conditional ablation of the ciliary assembly gene Ift20, an intraflagellar 

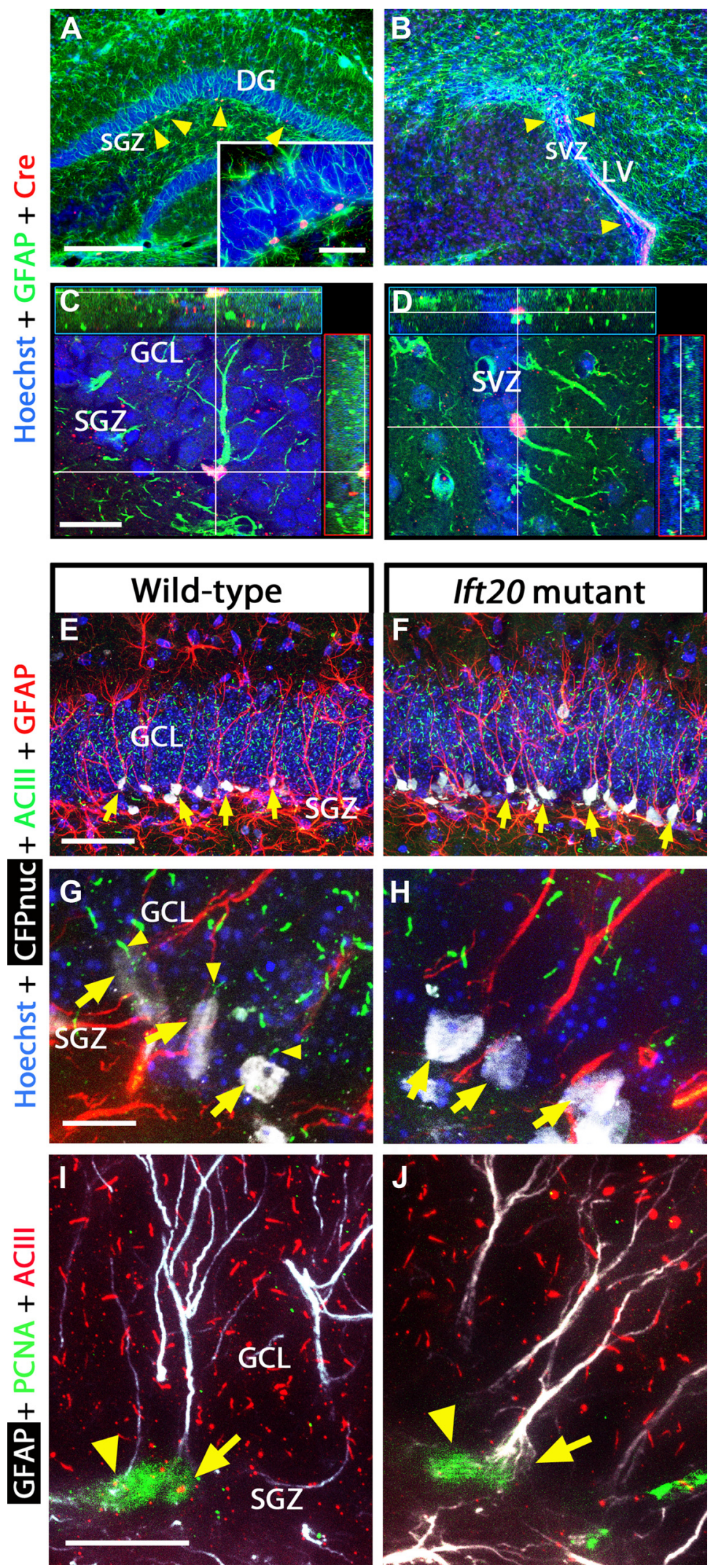

Figure 1. Primary cilia are ablated in GFAP-expressing NSCS from Ift2 $0^{\mathrm{fl} / f 1:}:$ mGFAP-Cre (Ift20 mutant) mice (Ift20 $0^{+/+}:$. mGFAP-Cre; wild type). Double immunostaining in 8-week-old Ift20 mutant mice shows (re expression (red) in $\mathrm{GFAP}^{+}$cells (green; arrowheads) in the SGZ (GCL, granule cell layer) of the hippocampal DG $(\boldsymbol{A})$ and in the SVZ of the LVs $(\boldsymbol{B})$. Confocal imaging showing GFAP ${ }^{+}$cells expressing Cre in the SGZ ( $\boldsymbol{C}$ ) and in the SVZ (D) of 8-week-old Ift20 mutant mice. Confocal imaging with CFPnuc (white), ACIII (green; marker for primary cilia), and GFAP (red) in the DG showing primary cilia and GFAPexpressing quiescent NSCS (and hence are nestin-CFPnuc ${ }^{+}$; arrows) from 8-week-old Ift22 ${ }^{+/+}$::mGFAP-Cre::nestin-CFPnuc transport (IFT) protein, results in loss of primary cilia and Shh signaling (Huangfu et al., 2003; Follit et al., 2006). By taking advantage of previously characterized transgenic mouse (mGFAP-Cre) in which the Cre recombinase protein is expressed in postnatal GFAP $^{+}$cells (Garcia et al., 2004), we ablated primary cilia in adult stem/progenitor cells. We show that postnatal primary cilia ablation results in a significant decrease of proliferation in hippocampal amplifying progenitors without affecting radial NSCs. Additionally, we show that chronically reduced level of adult hippocampal neurogenesis delays rather than permanently impairs spatial learning. Our results also indicate that decrease in hippocampal neurogenesis alters spatial novelty recognition and hippocampus-independent cue conditioning.

\section{Materials and Methods \\ Mice}

Generation of conditional Ift20 mutant mice was previously described (Jonassen et al., 2008). Briefly, Ift $20^{\text {flox }}$ mice were established by inserting loxP sites into intronic regions of Ift20 exons 1 and 3. Mice were bred with transgenic mice expressing Cre under control of the mouse glial fibrillary acidic protein promoter (mGFAP-Cre) (Garcia et al., 2004), resulting in $I f t 20^{\text {flox }}::$ mGFAP-Cre mice. Ift $20^{\text {flox }}::$ mGFAP-Cre mice were also bred with Z/EG double reporter mice (The Jackson Laboratory) and nestin-CFPnuc transgenic mice (Mignone et al., 2004), resulting in Ift $20^{\text {flox }}:$ :mGFAP-Cre::Z/EG and Ift $20^{\text {flox }}:$ :mGFAP-Cre::nestin-CFPnuc mice, respectively. All mice used in this study were fully backcrossed onto a C57BL/6 background. In all cases, mutant mice were directly compared with nontransgenic littermates. All mice were housed in a facility with a $12 \mathrm{~h}$ light/dark cycle and allowed ad libitum access to food and water. Experiments were conducted according to protocols approved by the Institutional Animal Care and Use Committee guidelines of Sanford-Burnham Medical Research Institute.

\section{$\leftarrow$}

(wild-type) (E) and /ft20 fl/fl:::mGFAP-Cre::"nestin-CFPnuc (Ift20 mutant) (F) mice. Confocal imaging with (FPnuc (white), ACIII (green), and GFAP (red) in the SGZ of the DG showing primary cilia (arrowheads) in GFAP-expressing radial NSCs (arrows) from 8-week-old lft20 $0^{+/+}:$:mGFAP-Cre::nestin-CFPnuc (wild type) $(\boldsymbol{G})$, whereas equivalent cells do not show primary cilia in Ift20 fl/fl:::mGFAP-Cre:::nestin-CFPnuc (Ift20 mutant) (H) mice. $I, J$, Confocal GFAP (white), PCNA (green), and ACIII (red) triple staining in DG of 8-week-old Ift20 f/fll:::mGFAP-Cre (Ift20 mutant) and wild-type mice. Adult hippocampal stem/progenitor cells in cell cycle $\left(\mathrm{PCNA}{ }^{+}\right)$show primary cilia staining $\left(\mathrm{ACIII}^{+}\right)$ in wild-type but not in Ift20 mutants [arrows, radial NSCs (radial GFAP ${ }^{+}$); arrowheads, other cells in cell cycle]. Section thickness: $A-J, 30 \mu \mathrm{m}$. Scale bars: $\boldsymbol{A}, \boldsymbol{B}, 200 \mu \mathrm{m} ; \boldsymbol{C}, \boldsymbol{D}, \boldsymbol{I}, \boldsymbol{J}, 20$ $\mu \mathrm{m} ; \boldsymbol{E}, \boldsymbol{F}, 50 \mu \mathrm{m} ; \boldsymbol{G}, \boldsymbol{H}, 10 \mu \mathrm{m}$. 


\section{Genotyping}

Specificity of Ift20 excision was evaluated by PCR using DNA from tail clip

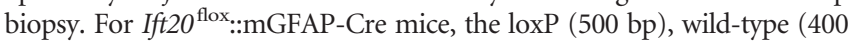
$\mathrm{bp})$, and Cre (200 bp) fragments were amplified simultaneously using four primers: 5'-ACTCAGTATGCAGCCCAGGT-3' (Ift20-F), 5' -GCTAGATGCTGGGCGTAAAG-3' (Ift20-R), 5' -CTGGCATTTCTGGGGATTGC-3' (Cre-F), and 5'-ACGGAAATCCATCGCTCGAC-3' (Cre-R). For

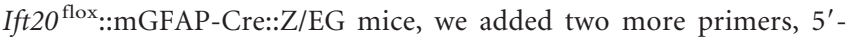
CTTGTACAGCTCGTCCATGC-3' (Z/EG-F) and 5'-ACGTAAACGGCCACAAGTTC-3' (Z/EG-R). For Ift20 flox::mGFAP-Cre::nestin-CFPnuc mice, we added two primers to the original set of four, $5^{\prime}$-GGAGCTGCACACAACCCATTGCC-3' (Nes-F) and 5'-GATCACTCTCGGCATGGACGAGC-3' (Nes-R). PCR was performed in $20 \mu \mathrm{l}$ reactions for 30 cycles using standard procedures; each cycle consisted of denaturing at $94^{\circ} \mathrm{C}$ for $30 \mathrm{~s}$, annealing at $60^{\circ} \mathrm{C}$ for $30 \mathrm{~s}$, and extension at $72^{\circ} \mathrm{C}$ for $45 \mathrm{~s}$, followed by a single 10 min extension at $72^{\circ} \mathrm{C}$. PCR products were analyzed in $2 \%$ agarose gels.

\section{BrdU}

For proliferation studies, 8-week-old and 12-month-old mice were given a single intraperitoneal injection of $\mathrm{BrdU}\left(100 \mathrm{mg} \mathrm{kg}^{-1}\right.$ body weight; $\mathrm{BD}$ Pharmingen; 51-7581KZ) followed by perfusion with $4 \%$ paraformaldehyde after $2 \mathrm{~h}$. To study adult neurogenesis, 8-week-old mice were injected for 5 consecutive days with $50 \mathrm{mg} \mathrm{kg}^{-1}$ body weight BrdU intraperitoneally twice daily $12 \mathrm{~h}$ apart and perfused with $4 \%$ paraformaldehyde 4 weeks after the last injection.

\section{Stereological cell quantification}

Cells positive for BrdU, NeuN, proliferating cell nuclear antigen (PCNA), Ki67, doublecortin (DCX), EGFP, CFPnuc, GFAP, and TUNEL were analyzed in serial sections through the DG of 8-week-old mice. We counted positive cells under a $63 \times$ objective using Slidebook software (Olympus). The total number of cells was counted using an optical dissector technique.

\section{Immunostaining}

Mice were perfused intracardially with $1 \times$ PBS followed by $4 \%$ paraformaldehyde. Brains were dissected and fixed overnight in $4 \%$ paraformaldehyde, rinsed, cryoprotected, and frozen in liquid $\mathrm{N}_{2}$. Cryosections (16 or $30 \mu \mathrm{m}$ ) were sliced on a cryostat. Standard immunostaining procedures were used for most antibodies, and appropriate conjugated secondary antibodies were used. For BrdU immunostaining, sections were pretreated in $2 \mathrm{~N} \mathrm{HCl}$ for $30 \mathrm{~min}$.

\section{Antibodies}

The following antibodies were used: rabbit anti-GFAP (1:500; Millipore; AB5804), mouse anti-NeuN (1:500; Millipore; MAB377), mouse antiNestin (1:500; Millipore; MAB353), chicken anti-GFP (1:500; Aves Lab; GFP-1020), guinea pig anti-DCX (1:1000; Millipore Bioscience Research Reagents; AB5910), mouse anti-Cre (1:400; Sigma-Aldrich; C7988), mouse anti-acetylated $\alpha$-tubulin (1:1000; Sigma-Aldrich; T6793), rabbit anti- $\gamma$-tubulin (1:1000; Sigma-Aldrich; T3359), rabbit anti-adenylyl cyclase III (1:500; Santa Cruz Biotechnology; sc-588), rat anti-BrdU (1:75; Serotec; OBT0030CX), mouse anti-PCNA (1:400; Dako; M0879), and rabbit anti-Ki67 (1:500; Vector; VP-RM04).

\section{TUNEL}

The Apoptag Red In Situ Apoptosis Detection Kit (S7165; Millipore Bioscience Research Reagents) was used in accordance with the manufacturer's protocol.

\section{Behavioral experiments}

Two different groups of mice were used, group $1[N=31$ mice ( 16 Ift $20^{\mathrm{f} / \mathrm{f}}:: \mathrm{mGFAP}-C r e$ and 15 wild type $\left.)\right]$ and group $2[N=21$ mice $(10$ Ift $20^{\mathrm{f} / \mathrm{f} l}:: \mathrm{mGFAP}-C r e$ and 11 wild type) $]$. Eight- to 12 -week-old male and female mice were acclimated to a reversed light cycle (on, 7:00 P.M.; off, 7:00 A.M.) housing room for 1 week before beginning behavioral testing, and all testing was done during the lights off (active) phase. Unless oth-
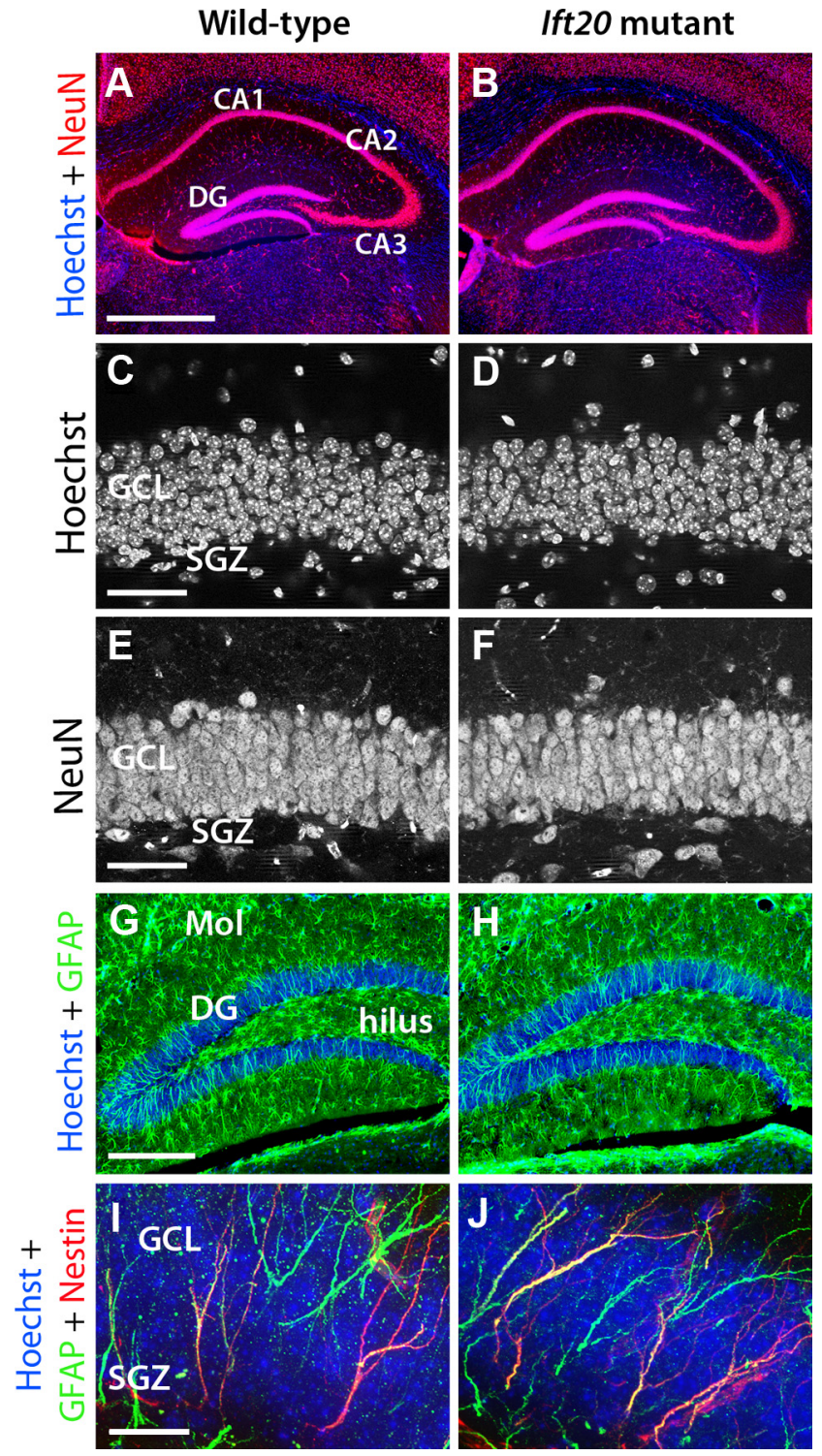

Figure 2. Neuromorphological characterization of the DG in 8-week-old Ift20 mutant (right panels) and wild-type (left panels) mice. $\boldsymbol{A}, \boldsymbol{B}$, NeuN (red) immunofluorescence shows preserved layering in hippocampal subregions (CA1, CA2, CA3, and DG) in Ift20 mutant and wildtype mice. $\boldsymbol{C}, \boldsymbol{D}$, Hoechst (white) nuclear staining in the $\mathrm{GCL}$ of the $\mathrm{DG}$ reveals no differences between Ift20 mutant and wild-type mice. $\boldsymbol{E}, \boldsymbol{F}$, NeuN (white) staining shows similar neuronal density in the $\mathrm{GCL}$ in both genotypes. $\mathbf{G}, \boldsymbol{H}$, Ift20 mutants show similar patterns of GFAP ${ }^{+}$ (green) astrocytes in the DG, hilus, and molecular layer (Mol) of the DG compared with wild-type mice. I, J, GFAP (green) and NESTIN (red) coexpression in cells from the SGZ of the DG indicates conserved morphology and distribution along the GCL. Section thickness: $A-J, 30 \mu \mathrm{m}$. Scale bars: $\boldsymbol{A}, \boldsymbol{B}, 500 \mu \mathrm{m} ; \boldsymbol{C}-\boldsymbol{F}, 50 \mu \mathrm{m} ; \boldsymbol{G}, \boldsymbol{H}, 200 \mu \mathrm{m} ; \boldsymbol{I}, \boldsymbol{J}, 20 \mu \mathrm{m}$.

erwise specified, tests were performed with mice from group 1 in the order below, separated by $5-10 \mathrm{~d}$.

\section{Locomotor activity test}

Locomotor activity was measured in polycarbonate cages $(42 \times 22 \times 20$ $\mathrm{cm})$ placed into frames $(25.5 \times 47 \mathrm{~cm})$ mounted with two levels of photocell beams at 2 and $7 \mathrm{~cm}$ above the bottom of the cage (San Diego Instruments). Mice were tested for $60 \mathrm{~min}$.

\section{Light/dark transfer test}

The light/dark transfer procedure measures anxiety-like behavior in mice (Crawley, 1999). A rectangular box made of Plexiglas is partitioned into two environments-one $(14.5 \times 27 \times 26.5 \mathrm{~cm})$ is dark $(8-16 \mathrm{lux})$ and the other $(28.5 \times 27 \times 26.5 \mathrm{~cm})$ is highly illuminated $(400-600 \mathrm{lux})$ by 
a $60 \mathrm{~W}$ light source located above it. The compartments are connected by an opening $(7.5 \times$ $7.5 \mathrm{~cm}$ ) located at floor level in the center of the partition. Mice were placed in the dark compartment to start the 5 min test.

\section{Novel object location tasks}

Spatial pattern recognition test. A procedure was adapted from that in the study by Benice and Raber (2008). Mice were individually habituated to a $51 \times 51 \times 39 \mathrm{~cm}$ open field for 5 min. For familiarization trials, three plastic toy objects (farmer boy, horse, and cow; Playmobil; Geobra Brandstatter) were placed in the open field (one in each of three corners). After three familiarization trials of $5 \mathrm{~min}$ each (separated by $1 \mathrm{~min}$ ), the mouse was tested in a location novelty recognition test in which one object was moved to a novel location in the arena. The same object was moved to the same location in all tests. Solving this test requires the ability to discriminate changes in pattern using proximal cues (i.e., movement relative to other objects in the field and relative to corners/sides).

Location novelty recognition test. Mice from group 2 were tested individually in an opaque Plexiglas disc $75 \mathrm{~cm}$ in diameter elevated $45 \mathrm{~cm}$ above the floor by a tripod. Distinct spatial cues kept constant throughout the study are located around the maze. For familiarization trials, a $250 \mathrm{ml}$ Erlenmeyer flask filled two-thirds with water (Pyrex) was placed in the same location, equidistant between the center and edge of the field. After four familiarization trials of $5 \mathrm{~min}$ each (separated by $1 \mathrm{~min}$ ), the mouse was tested in a location novelty recognition task in which the object was moved to a novel location in an arc of $45^{\circ}$, remaining at the exact same distance from the wall. In a previous pilot study, C57BL/6J mice showed that $45^{\circ}$ is the smallest degree change detection, as evidenced by increased exploration of the object after it was shifted. Solving this test requires the ability to discriminate changes in pattern using distal cues (i.e., movement only relative to cues outside the field as the relative position within the field appears unchanged).

Objects and the arena were thoroughly cleaned with $70 \%$ ethanol between trials to remove odors. The initial location was randomly assigned for each mouse such that all areas of the arena were used across testing. Numbers of object contacts were defined as touching the object with the nose.

\section{Cued and contextual fear conditioning}

Freezing responses are triggered by exposure to either the context in which shock was experienced (context test) or to the specific stimuli (cued test) associated with shock exposure. Conditioning took place in Freeze Monitor chambers (San Diego Instruments) housed in soundproof boxes. Conditioning chambers $(26 \times 26 \times 17 \mathrm{~cm})$ were made of Plexiglas with speakers and lights mounted on opposite walls. Chambers were installed with a shockable grid floor. On day 1 (habituation), mice were placed in the conditioning chamber for $5 \mathrm{~min}$ to habituate them to the apparatus. On day 2 (conditioning), the mice were exposed to the same context and conditioned stimulus (a tone plus light; 30 s, $3000 \mathrm{~Hz}$, $80 \mathrm{~dB}$ sound) in association with an unconditioned stimulus (footshock; $0.70 \mathrm{~mA}, 2 \mathrm{~s}$, scrambled current). A $5.5 \mathrm{~min}$ session was run in which mice received two shock exposures, both in the last $2 \mathrm{~s}$ of a $30 \mathrm{~s}$ tone plus light exposure. On day 3 (context test), contextual conditioning (as determined by freezing behavior) was measured in a 5 min test in the same chamber in which the mice were trained. On day 4 (cued test), mice were tested for cued conditioning. Mice were placed in a novel context (mod- ified context) for $3 \mathrm{~min}$ and then exposed to the conditioned stimulus (tone plus light) for $3 \mathrm{~min}$. For this test, the chamber was disguised with new walls, a new floor, and a novel odor.

To control for the order of context and cued testing and to avoid the potential extinction effects of the context test on the subsequent cued test, an additional group of mice (group 2) was conditioned identically, but tested first with the cues (on day 3 ) and then in the original context (on day 4). Freezing behavior was measured in all sessions by a validated computer-controlled recording of photocell beam interruptions.

\section{Optomotor test of vision}

This test uses a stationary elevated platform surrounded by a drum with black-and-white striped walls. The mouse is placed on the platform to habituate for $1 \mathrm{~min}$ and then the drum is rotated at $2 \mathrm{rpm}$ in one direction for $1 \mathrm{~min}$, stopped for $30 \mathrm{~s}$, and then rotated in the other direction for 1 min. The total number of head tracks $\left(15^{\circ}\right.$ movements at the speed of the drum) is recorded. Mice with intact vision track 6-12 times, whereas blind mice do not track at all.

\section{Barnes maze}

The procedure was adapted from that published by Bach et al. (1995). The Barnes maze is an opaque Plexiglas disc $75 \mathrm{~cm}$ in diameter elevated $58 \mathrm{~cm}$ above the floor by a tripod. Twenty holes, $5 \mathrm{~cm}$ in diameter, are located $5 \mathrm{~cm}$ from the perimeter, and a black Plexiglas escape box $(19 \times$ $8 \times 7 \mathrm{~cm}$ ) is placed under one hole. Distinct spatial cues kept constant throughout the study are located around the maze. On day 1, a training session consisting of placing the mouse in the escape box for $1 \mathrm{~min}$ was performed. One minute later, the mouse was placed in the middle of the maze and a buzzer $(80 \mathrm{~dB})$ and a light (400 lux) were turned on. The 


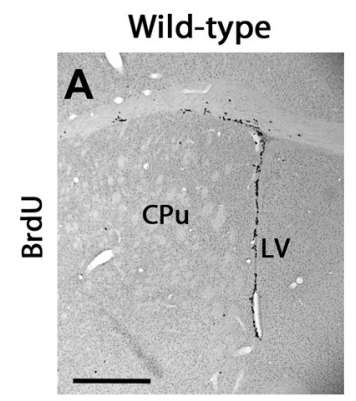

\section{Ift20 mutant}

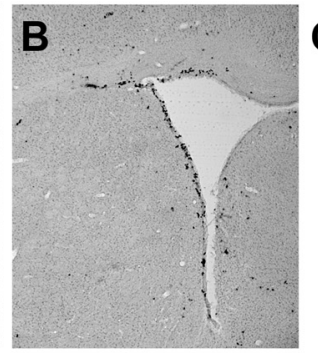

C
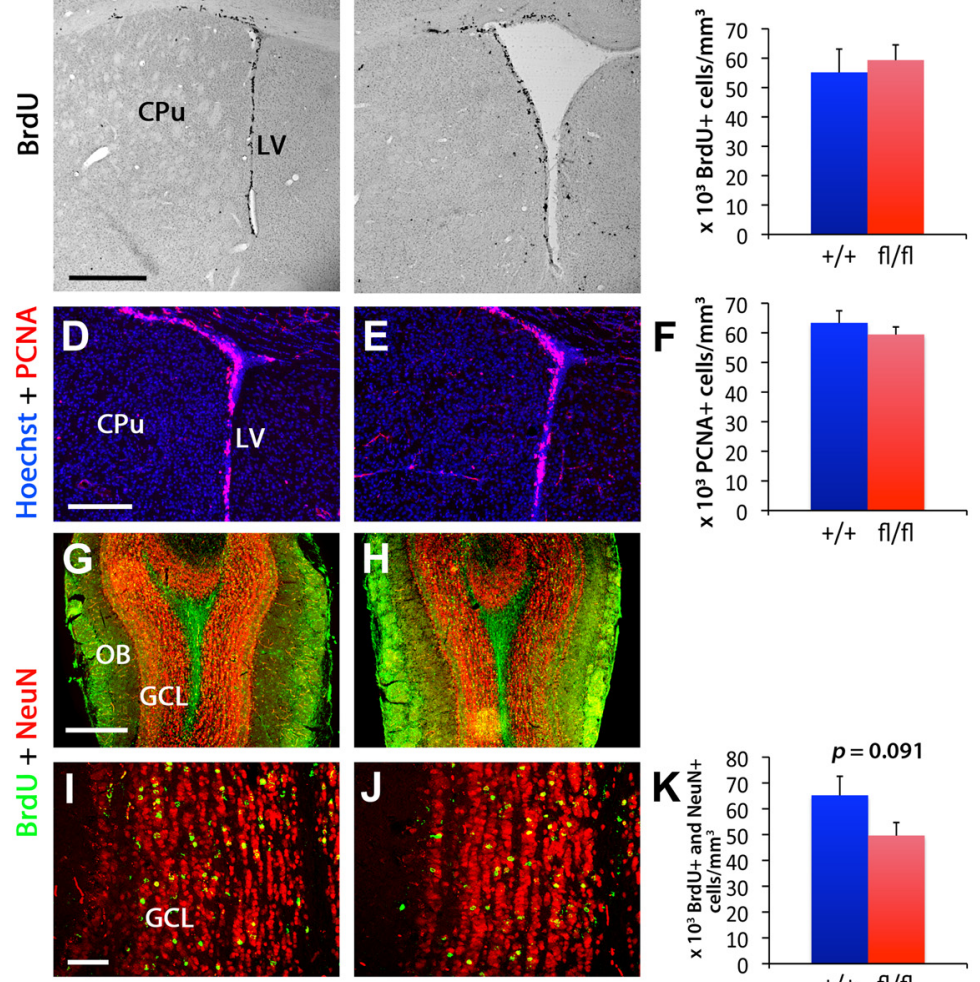

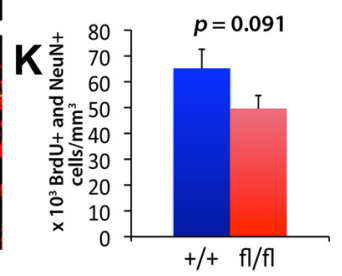

Figure 4. Proliferation in the SVZ of the LVs is similar in Ift20 mutant (right panels) and wild-type mice (left panels). $\boldsymbol{A}, \boldsymbol{B}$, BrdU staining in 8-week-old mice after a $2 \mathrm{~h} \mathrm{BrdU}$ pulse ( $100 \mathrm{mg} \mathrm{kg}^{-1}$ body weight) showed no evident genotypic differences in proliferation in the SVZ (CPu, caudate-putamen) (quantified in C). D, E, Staining with the proliferative marker PCNA (red) confirmed that proliferation in the SVZ is similar in Ift20 mutants and wild-type mice (quantified in $\boldsymbol{F}$ ). $\mathbf{G}, \boldsymbol{H}, \mathrm{A} 5 \mathrm{~d}$ BrdU injection protocol (twice daily, $12 \mathrm{~h}$ apart, at $50 \mathrm{mg} \mathrm{kg}^{-1}$ body weight) was used in 8-week-old Ift20 mutant and wild-type mice. Double labeling of BrdU (green) and NeuN (red) in the olfactory bulb (OB) 4 weeks after BrdU injection in Ift20 mutant and wild-type mice shows similar distribution of adult newborn neurons in the GCL in the two groups. I, J, BrdU (green) and NeuN (red) double staining in the $G C L$ of the $O B$ weeks after BrdU injection showed no genotypic differences in neurogenesis (quantified in $K$ ). Section thickness: $\boldsymbol{A}, \boldsymbol{B}, \boldsymbol{D}, \boldsymbol{E}, \boldsymbol{G}-\boldsymbol{J}, 30 \mu \mathrm{m}$. Scale bars: $\boldsymbol{A}, \boldsymbol{B}, 500 \mu \mathrm{m} ; \boldsymbol{D}, \boldsymbol{E}, \boldsymbol{G}, \boldsymbol{H}, 200 \mu \mathrm{m} ; \boldsymbol{I}, \boldsymbol{J}, 50 \mu \mathrm{m}$. Data are expressed as mean values \pm SEM.

session ended when the mouse entered the escape tunnel or after 3 min had elapsed. When the mouse did not enter the tunnel by itself, it was gently put in the escape box. Mice were allowed to remain in the escape tunnel for $1 \mathrm{~min}$. The tunnel was always located underneath the same hole, which was randomly determined for each mouse. Mice were tested once a day for $12 \mathrm{~d}$ for the acquisition portion of the study.

On the 13th day (probe test), the escape tunnel was removed and the mouse allowed to freely explore the maze for $3 \mathrm{~min}$.

Two weeks later, mice were retested with the escape box in the original position (retention test). On the day after this test, the escape tunnel was moved to a new location ( $90^{\circ}$ from the original position), and the behavior of the mouse was recorded as part of what is called the reversal test.

Sessions were videotaped and scored by an experienced observer blind to mouse genotype. Errors were defined as nose pokes and head deflections over any hole that did not have the tunnel beneath it. The count of errors that mice made in finding the target hole was modeled as a Poisson distribution. Search strategies were classified as follows: (1) random search, localized hole searches separated by crossings through the center of the maze; (2) serial search, systematic hole searches (every hole or every other hole) in a clockwise direction; (3) spatial search, reaching the escape tunnel with scores of $\leq 3$ for both errors and distance (number of holes between the first hole visited and the escape tunnel). An overall frequency was calculated for each type of strategy for each mouse.
$Y$-maze test for spontaneous alternations Spontaneous alternation on a three-armed $\mathrm{Y}$ maze is a model to study working memory. A single 5 min test was performed in which a mouse was placed in the center of the Y. Arm entries were recorded by video camera, and the total number of entries and their order were determined. Spontaneous alternations were defined as consecutive triplets of different arm choices. Normal mice tend to make between 60 and $70 \%$ spontaneous alternations of the total number of arm entries.

\section{Forced-swim behavior}

We modified the test originally described by Porsolt et al. (1977). Mice were individually placed into clear polypropylene cylinders containing $23-25^{\circ} \mathrm{C}$ water, $15 \mathrm{~cm}$ deep, for $6 \mathrm{~min}$. The time that each mouse was immobile, climbing the walls, or swimming was recorded by an observer blinded to genotype. The duration and latency for the first occurrence of each of the following behavioral items was taken as a dependent variable: (1) immobility, time spent by the mouse floating in the water without climbing and making only those movements necessary to keep its head above the water; (2) swimming, the animal displays active swimming motions displacing the body around the cylinder, more than necessary to merely keep the head above the water; (3) climbing, the animal vigorously moves all four legs with body aligned vertically in the water, usually directed against the wall of the cylinder.

\section{Statistical analysis}

One-way ANOVA and unpaired two-tailed $t$ tests were used to analyze histological data. One-way ANOVA was used to analyze behavioral tests. When necessary, data analysis was performed with repeated-measures ANOVA followed by post hoc comparisons with oneway ANOVA when appropriate (using SPSS 16.0 and $\mathrm{R}$ software). For all comparisons, values of $p<0.05$ were considered significant, and all data were presented as mean \pm SEM.

\section{Results}

\section{Deletion of primary cilia in adult NSCs in the DG}

Primary cilia are present in stem/progenitor cells and granule neurons in the adult hippocampal DG (Breunig et al., 2008). To conditionally ablate primary cilia in NSCs postnatally, we used previously described transgenic mice expressing Cre recombinase under control of the mGFAP promoter (Garcia et al., 2004). The Ift20 gene encodes an IFT protein essential for ciliogenesis (Follit et al., 2006). To target Ift20 in GFAP ${ }^{+}$cells, we engineered mGFAP-Cre mice carrying floxed Ift 20 alleles $\left(\right.$ Ift $20^{\mathrm{fl} / \mathrm{fl}}$ ) resulting in Ift $20^{\mathrm{fl} / \mathrm{fl}}:: \mathrm{mGFAP}-C r e$ (Ift 20 mutant) mice. Ift20 mutant mice were born at the expected Mendelian ratios and were indistinguishable from Ift $20^{+/+}:$:mGFAP-Cre (wild-type) littermates. The mGFAP promoter drives Cre recombinase expression in the hippocampal DG and in the lateral ventricles (LVs), giving rise to recombined cells (Fig. $1 A-D$ ). In this strain of mice, the mGFAPCre transgene in GFAP-expressing NSCs is not active during fetal development but is activated postnatally (Garcia et al., 2004), and a few detectable Cre-positive cells start to appear in the DG and LVs around postnatal day 7 (data not shown), evidencing that 
normal development of these brain regions are not affected. Isolated neural stem/progenitor cells from Ift20 mutants lacked primary cilia, whereas primary cilia were readily detectable in wild-type cells (data not shown). To verify that primary cilia are absent in radial NSCs in the DG of Ift20 mutant mice in vivo, we examined $\mathrm{GFAP}^{+} \mathrm{NESTIN}^{+}$cells using littermates of Ift 20 mutant mice crossed with mice that express a gene encoding a cyan fluorescent protein fused to a nuclear localization signal (CFPnuc) driven by the nestin enhancer (nestin-CFPnuc) (Encinas et al., 2006). The use of CFPnuc and GFAP expression helped to visualize radial NSCs in the DG. Confocal analysis with adenylyl cyclase III (ACIII), a marker for primary cilia, verified the lack of primary cilia in radial NSCs within the subgranule layer of DG in Ift20 mutant mice crossed to nestin-CFPnuc mice (Fig. $1 E-H$ ). In addition, by staining for the proliferative cell marker PCNA, we confirmed that radial NSCs and amplifying progenitors in cell cycle in Ift20 mutants do not have primary cilia (Fig. 1 $I, J$ ).

\section{Normal morphology of brain structures in adult Ift20 mutant mice}

We conducted several experiments to detect potential morphological abnormalities in the brain structures after postnatal deletion of Ift 20 gene. At 8 weeks of age, the Ammon's horn of the hippocampus in Ift20 mutants exhibited normal morphology (Fig. 2A,B). Moreover, the DG of these mice showed normal organization and was the same size as that seen in wildtype mice (Fig. 2C,D). Both in wild-type and Ift 20 mutant mice, staining using the neuronal marker NeuN revealed numerous nuclei of cells corresponding to mature neurons representing granule cells, without any evidence of altered density (Fig. 2 E, F ). In addition, the GFAP ${ }^{+}$astrocytes in the DG, hilus, molecular layer, and the remainder of the hippocampus were present in Ift20 mutant mice in similar numbers and with similar morphologies as in wild-type mice (Fig. 2G,H).

The SGZ contains a heterogeneous population of stem/progenitor cells. One type is the radial NSCs (or type 1 cells), which are relatively quiescent, show morphologies characteristic of radial glia (Seri et al., 2001), and express the precursor cell marker protein NESTIN and the astrocytic protein GFAP (Seri et al., 2004). To determine whether the organization of the radial $\mathrm{GFAP}^{+} / \mathrm{NESTIN}^{+}$NSCs was defective in the mutant DG, we costained for GFAP and NESTIN and observed a similar pattern of radial processes in both genotypes (Fig. $2 I, J$ ). This finding indicates that the lack of cilia in radial NSCs does not appreciably alter their morphology of radial astrocytes in the SGZ.

Other brain regions that undergo considerable postnatal neurogenesis-including the cerebellar GCL and the olfactory bulb (OB) (data not shown)—showed normal size, morphology, and density of mature neurons in 8-week-old Ift20 mutant mice, suggesting that neurons in these regions had already formed before

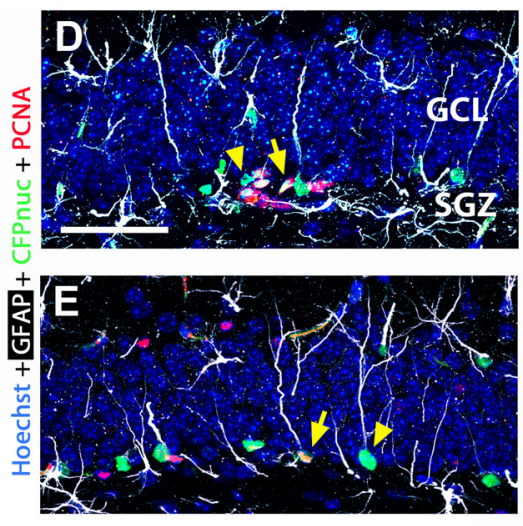

$\mathbf{F}$

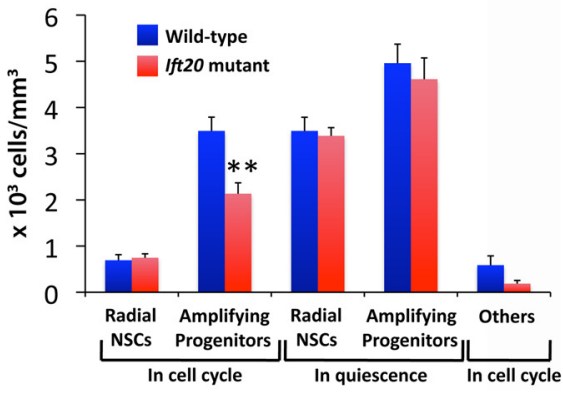

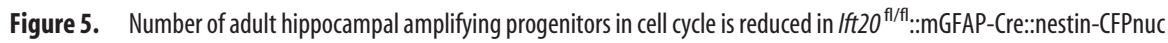
(Ift20 mutant) mice. $\boldsymbol{A}, \boldsymbol{B}$, Confocal GFAP (red) and CFPnuc (green) double staining in DG of 8-week-old

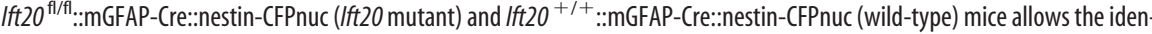
作 PCNA ) and in quiescence (arrowhead; PCNA $^{-}$). $\boldsymbol{F}$, Quantification of SGZ stem/progenitor cells (CFPnuc ${ }^{+}$) in 8-week-old

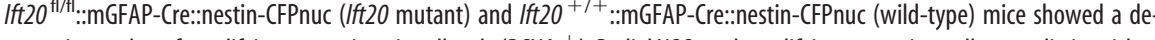
列 by the presence or lack of a radial GFAP ${ }^{+}$process, respectively. Section thickness: $A, B, D, E, 30 \mu \mathrm{m}$. Scale bars: $A, B, 100 \mu \mathrm{m} ; \boldsymbol{D}$, $\boldsymbol{E}, 25 \mu \mathrm{m} .{ }^{* *} p<0.01$. Data are expressed as mean values \pm SEM.

the conditional deletion of Ift20 affects primary cilia formation in the mutant mice.

\section{Decreased adult hippocampal cell proliferation in Ift20} mutant mice

Ift20 mutant mice show gradual decrease in SGZ proliferation with a significant reduction already at 4 weeks of age (data not shown). To evaluate the proliferation of stem/progenitor cells in the SGZ of 8-week-old Ift20 mutant mice, we injected mice with the cell cycle marker BrdU. A $2 \mathrm{~h}$ pulse of BrdU $\left(100 \mathrm{mg} \mathrm{kg}^{-1}\right.$ body weight) resulted in many labeled cells in the SGZ of wildtype mice (Fig. $3 A$ ). By contrast, the number of BrdU-labeled cells in the DG of Ift 20 mutants was $54.5 \%$ of the number in wild-type mice $\left(5.51 \pm 0.8\right.$ vs $10.11 \pm 1.27 \times 10^{3}$ cells $/ \mathrm{mm}^{3} ; p=$ 0.006 ) (Fig. $3 A-C$ ). The number of cells in the SGZ positive for the proliferative cell marker PCNA was similarly reduced $(39.2 \%$ of wild type; $3.96 \pm 0.54$ vs $10.1 \pm 0.81 \times 10^{3}$ cells $/ \mathrm{mm}^{3} ; p<$ 0.000001 ) (Fig. $3 D-F$ ). These data indicate that proliferation is reduced approximately twofold in the SGZ of Ift 20 mutant mice. To assess whether reduction in proliferation was maintained in Ift20 mutants at older stages, we analyzed 12-month-old mice. The number of Ki67-labeled cells in the DG was similarly reduced (61.5\% of wild type; $0.51 \pm 0.08$ vs $0.84 \pm 0.13 \times 10^{3}$ cells $/ \mathrm{mm}^{3}$; $p=0.035)$ at this stage. 

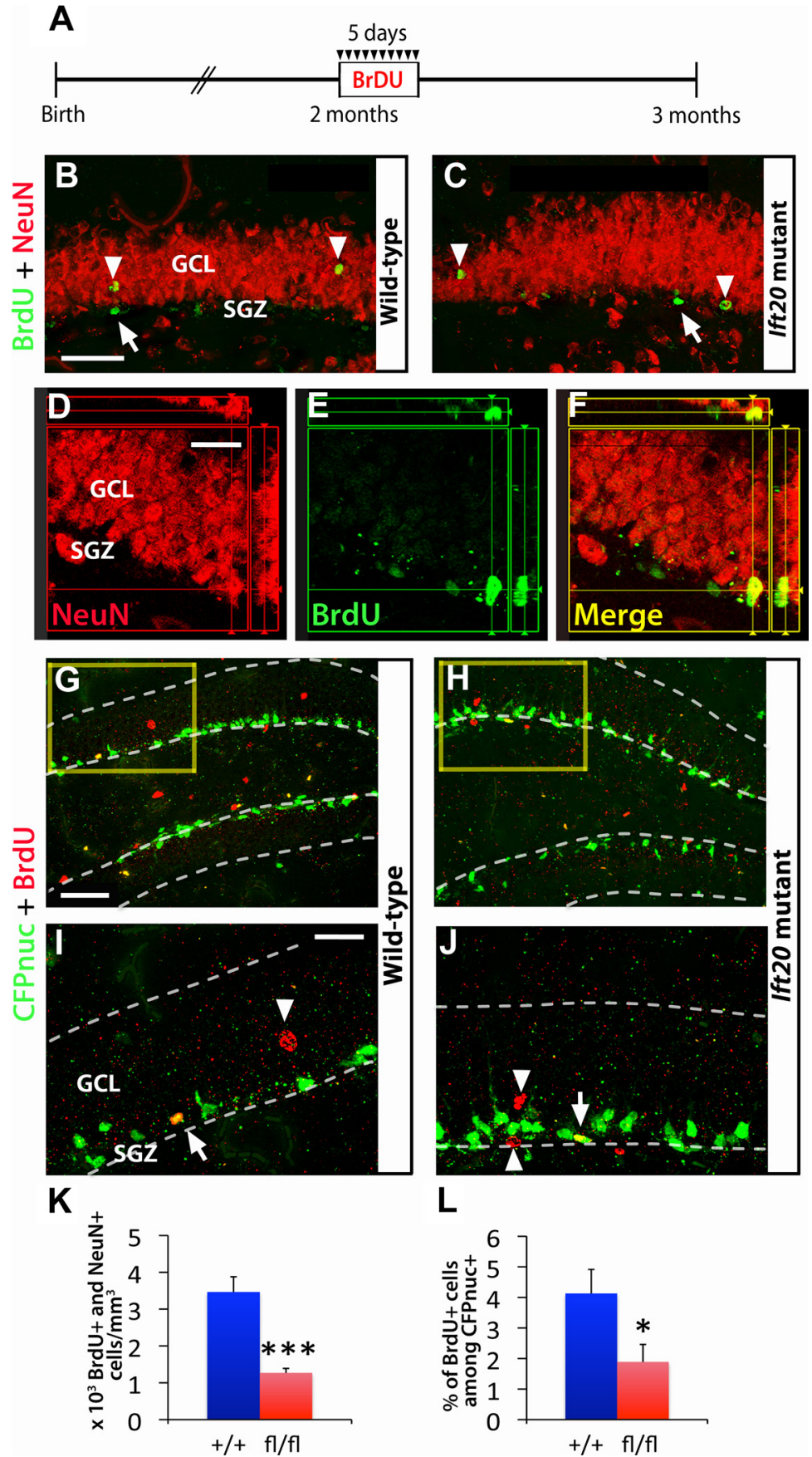

L

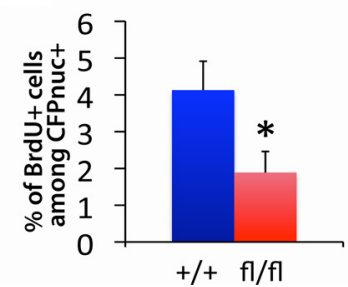

Figure 6. Adult hippocampal neurogenesis is reduced in Ift20 mutant mice. $A, A 5 \mathrm{~d}$ BrdU injection protocol (twice daily, $12 \mathrm{~h}$ apart, at $50 \mathrm{mg} \mathrm{kg}^{-1}$ body weight) was used in 8-week-old /ft20 f//fl::mGFAP-Cre (Ift20 mutant) and /ft20 ${ }^{+/+}$::mGFAP-Cre (wild-type) mice, and the BrdU-labeled cells were examined 4 weeks after the last injection. B, C, BrdU (green) and NeuN (red) double staining 4 weeks after BrdU injection in /ft20 mutant and wild-type mice reveals BrdU-labeled adult newborn neurons (arrowheads; $\mathrm{NeuN}^{+}$) as well as other cell types in the SGZ (arrows; NeuN ${ }^{-}$). D-F, Colocalization of a BrdU ${ }^{+}$(green) cell that coexpress the neuronal marker NeuN (red). G, H, CFPnuc (green) and BrdU (red) double staining, 4 weeks after BrdU injection, in 8-week-old /ft20 f//fl.:mGFAP-Cre::nestin-CFPnuc (Ift20 mutant) and Ift20 ${ }^{+1+}:$ :mGFAP-Cre::nestin-CFPnuc (wild-type) mice showing colocalization of BrdU ${ }^{+} / \mathrm{CFPnuc}^{+}$cells along the SGZ. Higher magnification (squares) of $\boldsymbol{G}$ and $\boldsymbol{H}$ are displayed in I and $\boldsymbol{J}$, respectively (arrows, BrdU ${ }^{+}$stem/progenitor cells; arrowheads, other BrdU ${ }^{+}$cells). $\boldsymbol{K}$ Quantification of BrdU ${ }^{+} / \mathrm{NeuN}^{+}$cells showed reduced neurogenesis in the $\mathrm{GCL}$ of the DG in Ift20 mutants compared with wild-type mice. $L$, Quantification of the percentage of BrdU ${ }^{+}$among CFPnuc ${ }^{+}$cells indicated reduced numbers of stem/progenitor cells that retained BrdU in Ift20 mutants compared with wild-type mice. Section thickness: $\boldsymbol{B}-\boldsymbol{J}, 30 \mu \mathrm{m}$. Scale bars: $\boldsymbol{B}, \boldsymbol{C}, 100 \mu \mathrm{m} ; \boldsymbol{D}-\boldsymbol{F}, \boldsymbol{I}, \boldsymbol{J}, 25 \mu \mathrm{m} ; \boldsymbol{G}, \boldsymbol{H}, 50 \mu \mathrm{m}$. ${ }^{*} p<0.05 ;{ }^{* * *} p<0.001$. Data are expressed as mean values \pm SEM.

A reduced number of proliferating cells in the DG of Ift20 mutants should decrease the number of neuroblasts. In agreement with this prediction, immunostaining with the neuroblast marker DCX revealed a reduced number of neuroblasts in the DG of 8-week-old $(57.5 \%$ of wild type; $30.32 \pm 4.48$ vs $52.73 \pm 4.33 \times 10^{3}$ cells/ $\mathrm{mm}^{3} ; p<0.001$ ) (Fig. 3G-I) and 12month-old $(62.1 \%$ of wild type; $4.42 \pm$ 0.29 vs $7.12 \pm 0.59 \times 10^{3}$ cells $/ \mathrm{mm}^{3} ; p<$ 0.0001) Ift20 mutant mice.

Newborn cells that are not recruited into functional circuits in the adult DG are eliminated by apoptotic cell death (Biebl et al., 2000). To analyze whether the reduction in proliferating/ $\mathrm{DCX}^{+}$cells is due to apoptosis in the SGZ, we performed immunostaining for TUNEL $^{+}$ cells. We observed similar numbers of apoptotic cells in the SGZ of Ift20 mutants and wild-type mice (Fig. 3J-L).

Despite the fact that ablation of primary cilia in neural precursor cells of the adult SGZ significantly reduced neurogenesis, 8-week-old Ift20 mutant mice did not showed changes in proliferation in the subventricular zone (SVZ) of the LVs, as evidenced by BrdU incorporation and PCNA staining analyses (Fig. $4 A-F$ ). This observation was confirmed by DCX staining, which revealed that the density of neuroblasts in the SVZ was similar in Ift20 mutant and wild-type mice (data not shown). Further analysis at older stages (12 months of age) revealed a modest reduction in number of BrdU-labeled cells ( $83.7 \%$ of wild type; $24.47 \pm 1.49$ vs $29.24 \pm 1.65 \times 10^{3}$ cells $/ \mathrm{mm}^{3} ; p=0.037$ ) in the SVZ of Ift20 mutant mice.

\section{Primary cilia regulate proliferation of} hippocampal amplifying progenitors To analyze the numbers of radial NSCs and amplifying progenitors in the SGZ of Ift20 mutants we used 8 -week-old Ift20 ${ }^{+/+}:$: mGFAP-Cre::nestin-CFPnuc mice. Staining for GFAP, an intermediate filament protein organized in the processes traversing granule layer in radial NSCs (type 1 cells), and for CFPnuc, which is localized to nuclei of cells expressing NESTIN (type 1 and type 2a cells) (Fig. 5A,B), allowed us to distinguish between radial NSCs (CFPnuc ${ }^{+}$cells with radial $\mathrm{GFAP}^{+}$processes) and amplifying progenitors $\left(\mathrm{CFPnuc}^{+}\right.$cells without $\mathrm{GFAP}^{+}$radial processes). Total numbers of radial NSCs were similar in both genotypes (Fig. $5 C$ ), suggesting that the lack of primary cilia does not affect self-renewal and/or survival on this cell population. In contrast, the numbers of amplifying progenitors were significantly reduced in Ift 20 mutants (79.8\% of wild type; $6.74 \pm 0.53$ vs $8.45 \pm$ $0.26 \times 10^{3}$ cells $/ \mathrm{mm}^{3} ; p<0.01$ ) (Fig. $5 C$ ), indicating that primary cilia ablation specifically affected this cell population.

To determine how lack of primary cilia affects proliferation in radial NSCs and amplifying progenitors, we used staining for 
PCNA in combination with staining for GFAP and CFPnuc (Fig. 5D,E). Analysis of radial NSCs showed no difference between genotypes within both quiescent $\left(\mathrm{PCNA}^{-} \mathrm{CFPnuc}^{+}\right.$radial $\mathrm{GFAP}^{+}$) and proliferating $\left(\mathrm{PCNA}^{+} \mathrm{CFPnuc}^{+}\right.$radial $\mathrm{GFAP}^{+}$) subpopulations (Fig. $5 F$ ). Quiescent amplifying progenitors (PCNA ${ }^{-}$ $\mathrm{CFPnuc}^{+}$) showed no differences between genotype; however, the numbers of proliferating amplifying progenitors $\left(\mathrm{PCNA}^{+} \mathrm{CFPnuc}^{+}\right.$) were significantly reduced in Ift 20 mutants $(61.1 \%$ of wild type; $2.13 \pm 0.24$ vs $3.49 \pm 0.29 \times 10^{3}$ cells $/ \mathrm{mm}^{3}$; $p<0.01)$. These results indicate that the loss of primary cilia in the DG specifically reduced the numbers of proliferating, but not quiescent, amplifying progenitors without affecting the numbers of either proliferating or quiescent radial NSCs.

In the SVZ, analysis of stem/progenitor cells stained for CFPnuc in 8-week-old mice did not reveal major differences between mutant and wildtype mice (data not shown). These findings suggest that the lack of primary cilia in neural precursors does not affect the overall cell numbers in this neurogenic niche.

\section{Reduced neurogenesis in the DG of Ift20 mutant mice}

It remains unknown whether loss of cilia in adult NSCs would alter adult neurogenesis. To analyze the proportion of neurons that mature and integrate into the DG, we injected BrdU into 8 -week-old mice for $5 \mathrm{~d}$ (twice daily, $12 \mathrm{~h}$ apart, at $50 \mathrm{mg} \mathrm{kg}^{-1}$ body weight) and examined the BrdU/NeuN-labeled cells 4 weeks after the last injection (Fig. 6A,D-F). The number of $\mathrm{BrdU}^{+} / \mathrm{NeuN}^{+}$cells in Ift 20 mutant mice was $36.6 \%$ of that seen in wild-type mice $\left(1267.9 \pm 123.2\right.$ vs $3465.3 \pm 413.2$ cells $/ \mathrm{mm}^{3}$; $p<0.001$ ) (Fig. 6B, $C, K$ ). Independently, we also analyzed the cumulative numbers of postnatal newborn neurons integrated in the DG of Ift20 mutants. Crossing with mice harboring a reporter designated Z/EG (lacZ/Lox-Stop/EGFP) (Novak et al., 2000), in which postnatal Cre recombination activates expression of an EGFP reporter, allowed lineage tracing of stem/progenitor progeny. Double staining for NeuN and EGFP in 8-week-old Ift $20^{\mathrm{fl} / \mathrm{fl}}:: \mathrm{mGFAP}-C r e:: \mathrm{Z} / \mathrm{EG}$ (Ift20 mutant) mice and Ift $20^{+/+}$: mGFAP-Cre::Z/EG (wild-type) mice showed decrease in numbers of that $\mathrm{EGFP}^{+} \mathrm{NeuN}^{+}$double-positive cells in the DG of Ift 20 mutant mice $(31.9 \%$ of wild type; $147.8 \pm 26.9$ vs $462.5 \pm$ $35.3 \times 10^{3}$ cells $\left./ \mathrm{mm}^{3} ; p<0.001\right)$. These data indicate that the cumulative ( 8 week) decrease in adult neurogenesis (approximately threefold) is similar to the neurogenesis rate measured over $5 \mathrm{~d}$ of BrdU incorporation, suggesting that the reduction in generation of new neurons in Ift20 mutants is constant over time.

Colocalization of EGFP and NeuN markers suggested that neurons derived from radial NSCs that had undergone primary cilia ablation were present in the GCL of Ift20 mutants (data not shown). In addition, a stronger reduction in the numbers of granule cells (approximately threefold) compared with $\mathrm{DCX}^{+}$cells (approximately twofold) hints at a potential role for primary cilia in the survival of $\mathrm{DCX}^{+}$neuroblasts.

The presence of $\mathrm{BrdU}^{+} \mathrm{NeuN}^{-}$cells in the SGZ suggested that some non-neuronal cells could retain BrdU for 4 weeks. We analyze the percentage of CFPnuc ${ }^{+}\left(\mathrm{NESTIN}^{+}\right)$stem/ progenitor cells that retain $\mathrm{BrdU}^{+}$after 4 weeks in Ift $20^{\mathrm{fl} / \mathrm{fl}}:$ :
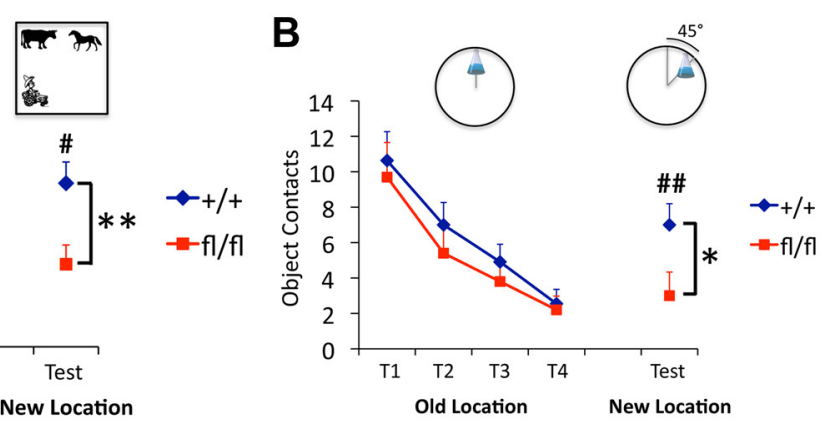

mGFAP-Cre::nestin-CFPnuc (Ift20 mutant) mice. Compared with wild-type mice, Ift20 mutants show approximately twofold decrease in numbers of stem/progenitor cells that retain $\mathrm{BrdU}^{+}$ after 4 weeks ( $45.8 \%$ of wild-type; $1.89 \pm 0.57$ vs $4.13 \pm 0.78 \%$ of $\mathrm{BrdU}^{+}$cells among CFPnuc ${ }^{+}$cells; $p<0.001$ ) (Fig. 6G-J,L). This decrease is similar to that seen in global proliferation studies, suggesting that the survival of stem/progenitor cells that had previously divided is similar in Ift20 mutants and wild-type mice.

Analysis of $\mathrm{BrdU}^{+} \mathrm{NeuN}^{+}$cells in the OB 4 weeks after the last BrdU injection did not show differences between genotypes (Fig. $4 G-K$ ). Furthermore, $\mathrm{EGFP}^{+}$cells in the OB of 8 -week-old Ift $20^{\mathrm{fl} / \mathrm{fl}}:: \mathrm{mGFAP}-\mathrm{Cre}: \mathrm{Z} / \mathrm{EG}$ mice derived from recombined $\mathrm{GFAP}^{+}$NSCs in the LVs did not show any major defect in neurogenesis (data not shown). These results indicate that adult neurogenesis in the SVZ-OB is not significantly affected in Ift20 mutant mice, consistent with the previous finding that primary cilia in NSCs are largely dispensable for the development of the SVZ (Han et al., 2008).

\section{Ift20 mutant mice show altered spatial novelty recognition}

One of the most striking challenges in the field of adult hippocampal neurogenesis is to elucidate a causal relationship between neurogenesis and learning and memory. To evaluate spatial recognition in Ift20 mutant mice, we used two different novel location-object recognition tests, a spatial pattern recognition test and a location novelty recognition test. Briefly, in the spatial recognition test, mice were habituated to three different objects in an open field. After familiarization trials, they were tested in a novelty recognition test in which one object was moved to a novel location in the arena. We found no genotypic difference in habituation to test objects across three familiarization trials: mice of both genotypes made fewer contacts with the objects over time (Fig. 7A). In contrast, when one of the objects was moved to a novel location, there were striking effects on reexploration of the displaced object, in which a renewed interest (increased contacts) in the object was observed in wild-type $(p<$ 0.05 ) but not in Ift20 mutant mice. Post hoc analysis revealed significant differences between genotypes in reexploring the displaced object $(p<0.01)$.

The initial spatial pattern recognition test relies on the ability of the animals to detect a change in the spatial patterning of 
A

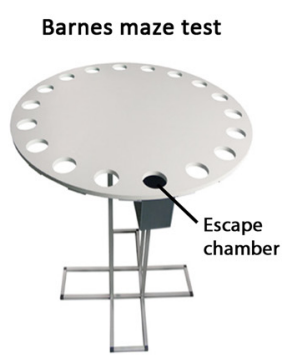

B

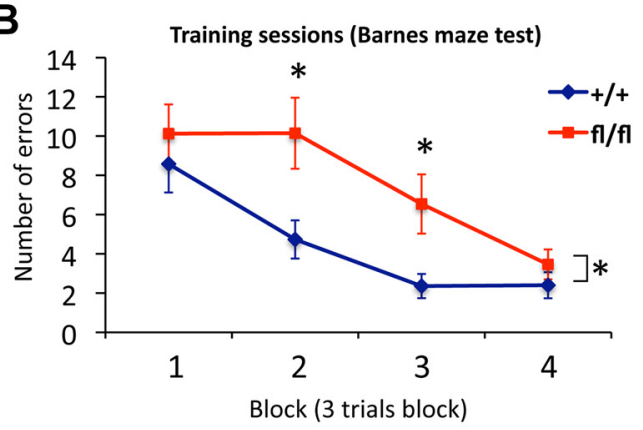

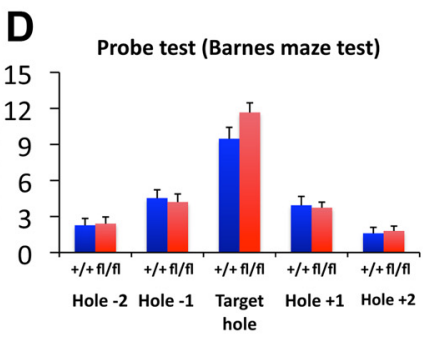

$\mathrm{E}$

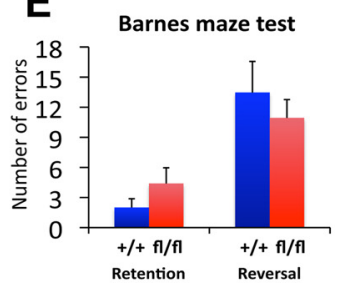

$\mathbf{F}$

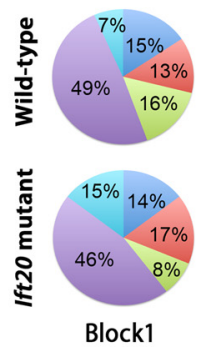

Search strategies (Barnes maze test)
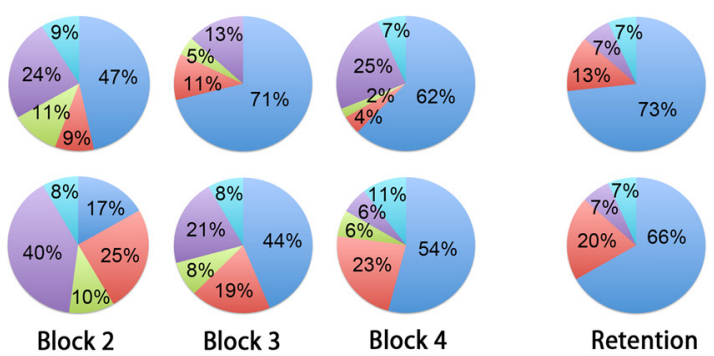

Figure 8. Ift20 mutant mice exhibit delayed spatial learning but preserved long-term memory. $\boldsymbol{A}$, Schematic representation of Barnes maze test. $\boldsymbol{B}$, In the Barnes maze, Ift20 mutants showed delayed spatial learning in finding the escape chamber (averaged into 4 blocks of 3 trials) but reached similar levels on task performance in the last block. $C, D$, Ift20 mutants and wild-type mice spent similar amount of time in the target quadrant $(\boldsymbol{C})$ and made similar numbers of head deflections at the target hole during the probe test (no escape chamber) of the Barnes maze (D), showing that both groups had learned the original position of the chamber. $\boldsymbol{E}$, Ift20 mutants showed normal retention and reversal of the task 2 weeks later. $F$, Ift20 mutants exhibited a delay in using a spatial strategy as evidenced by an increase in use of this strategy at the third block, whereas wild-type mice begin using a spatial strategy at the second block. Two weeks after the last trial (retention test), no differences in the use of spatial strategy were observed between the two genotypes. Shown is the percentage of each search strategies used by Ift20 mutants and wild-type mice across blocks (averaged into 4 blocks of 3 trials) and retention test on the Barnes maze (values represent group means). Search strategies are defined as follows: spatial, reaching the escape tunnel with scores of $\leq 3$ for both error and distance (number of holes between the first hole visited and the escape tunnel); nonspatial, serial, random, random/serial, and other strategies are described in Materials and Methods. $N=31$ mice ( $16 \mathrm{lft} 20^{\mathrm{fl} / \mathrm{fl}:}:$ :mGFAP-Cre and 15 wild-type). ${ }^{*} p<0.05$. Data are expressed as mean values \pm SEM.

objects relative to each other. In the location novelty recognition test, a more complex object exploration task, mice are tested for their ability to detect the displacement of a single object moved $45^{\circ}$ in an arc around a circular field. Success requires the mice to use distal spatial cues to discriminate similar contexts. We found no genotypic difference in habituation to test the object across four familiarization trials (Fig. 7B). When the object (Erlenmeyer flask) was moved to a novel location, a significant renewed interest (increased contacts) in the object was observed in wild-type $(p<0.01)$ but not in Ift20 mutant mice. Post hoc analysis revealed significant differences between genotypes in reexploring the displaced object $(p<0.05)$. These results indicate that subtle changes in spatial context are not detected by the mutant mice and suggest decreased efficiency or impairment in spatial novelty recognition.

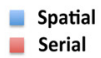

- Serial

Random/Serial

- Random

Other

\section{Ift20 mutant mice show delay in spatial learning}

As a further more complex test of spatial learning, we used the Barnes maze, which consists of an elevated maze with 20 holes (Fig. 8A). An escape chamber is located in one of the holes, and mice learn to locate the chamber over $12 \mathrm{~d}$, in four blocks of three trials. Using repeated-measures ANOVA of the numbers of errors to find the target hole, we found the interaction term between blocks and genotype to be significant $(p<0.05)$ (Fig. $8 B)$, in that mutant mice performed poorly in the first blocks. Overall, Ift20 mutant mice made more errors in locating an escape chamber and ultimately escaping. Closer analysis showed that the difference was significant in blocks 2 and $3(p<0.05)$. A day later (following the 12 trials), when we performed a probe test in which the escape chamber was removed and time spent in the target quadrant and hole search strategies was assessed, the two groups of mice performed similarly (Fig. 8C,D). Analysis undertaken 2 weeks after the last trial (retention trial) showed that long-term memory was not affected (Fig. $8 E$ ). In addition, mice exposed to a reversal trial (in which the escape chamber was moved to a new location) did not show delayed learning, illustrating that the working memory strategies adopted by the mice to locate a new escape chamber location were preserved. A comparison of strategies used by wild-type versus mutant mice also revealed significant differences (Fig. $8 F$ ). Wild-type mice progressively increased the use of a spatial strategy across the first three blocks, whereas mutant mice were slower to shift to a spatial strategy and instead relied on random and/or serial strategies for longer periods. However, the spatial strategy used in Ift20 mutant mice in the last block of trials and in the retention trial was similar to that used by wildtype mice. Thus, Ift20 mutant mice could learn a spatial contingency but required more trials to do so, suggesting that they have delayed rather than permanently impaired spatial learning.

Additional behavioral tasks were performed to test for locomotor dysfunction, visual disabilities, lack of motivation, anxiety-related and depression-like behaviors (data not shown) with no differences between genotype.

\section{Ift20 mutant mice show enhanced cued fear responses}

The link between adult hippocampal neurogenesis and contextual fear learning, a form of pavlovian conditioning, is still unclear (Shors et al., 2002; Saxe et al., 2006; Dupret et al., 2008; Imayoshi et al., 2008).

Contextual and cued fear learning are induced by pairing neutral conditioned stimuli (tone plus light) with an aversive unconditioned stimulus (electric shock) in a specific context, and the 
A

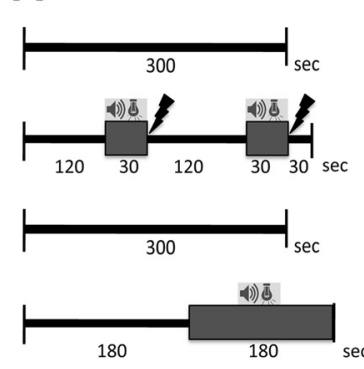

B

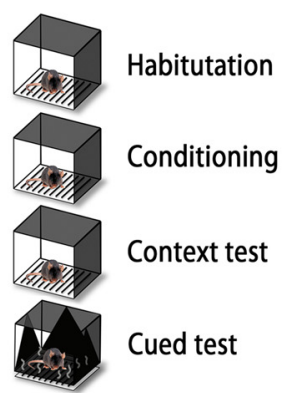

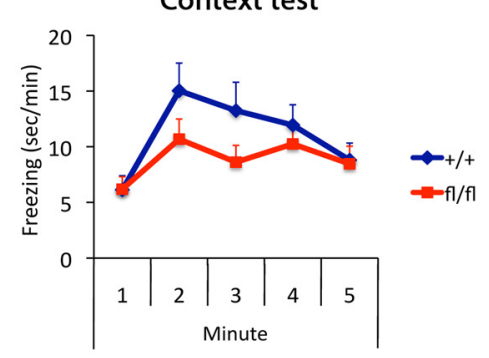

C

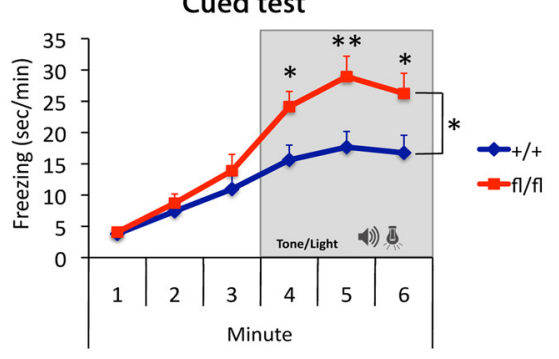

Figure 9. Ift20 mutant mice exhibit enhanced fear responses to cued but not contextual fear conditioning. $A, A$ d fear conditioning protocol was used to evaluate contextual and cued fear conditioning. $\boldsymbol{B}$, The freezing response to the same context than conditioning does not differ between the two genotypes (context test, mice group 1 and 2 ). $\boldsymbol{C}$, Ift20 mutants show a higher freezing response during the exposition to the tone plus light combination (cue) in a different context (cued test, mice groups 1 and 2). Group 1, Thirty-one mice (16 Ift20 fl/fl.::mGFAP-Cre and 15 wild type); group 2, 21 mice (11 Ift20 fl/fl::mGFAP-Cre and 10 wild type). ${ }^{*} p<0.05 ;{ }^{* *} p<0.01$. Data are expressed as mean values \pm SEM.

extent of "freezing" behavior is used as an index of effective retrieval of the consolidated fear memory. Following pairing of a shock with a tone plus light combination in a specific context (conditioning), mice were tested in the same context without shock or tone plus light combination (context test) (Fig. 9A). Ift20 mutants showed slightly reduced patterns of freezing behavior than wild-type mice across the context test (nonsignificant) (Fig. 9B). In addition, mice were tested in a different context with the introduction of the tone plus light combination (cued test). Interestingly, Ift20 mutants showed higher levels of freezing during the exposure to the tone plus light combination $(p<0.05$ and $p<0.01$ ) (Fig. 9C), but not before, indicating that Ift 20 mutants responded more robustly to the cue than wild-type mice.

The greater levels in freezing behavior of Ift20 mutants after the introduction of the neutral stimuli (tone plus light) in a different context suggests that Ift20 mutant mice are biased toward cue-based strategies.

\section{Discussion}

Primary cilia regulate proliferation in hippocampal amplifying progenitors

Primary cilia and Shh signaling in NSCs during development are required for the formation and expansion of the adult hippocampal NSC pool (Breunig et al., 2008; Han et al., 2008). Although detection of Shh signaling in adult NSCs (Ahn and Joyner, 2005) suggests its critical role in the homeostatic regulation of adult neurogenesis, our understanding of the molecular mechanisms is still very limited.

We have addressed the role of primary cilia in the adult hippocampal stem cell population by conditional deletion of Ift 20 (Jonassen et al., 2008), an IFT protein required for cilia formation and Shh signaling (Huangfu et al., 2003; Follit et al., 2006). Contrary to cilia deletion during development, postnatal deletion of cilia in neural stem/progenitor cells did not affect the number of radial NSCs (type 1 cells) or proliferation of this cell population in adult DG. Instead, primary cilia ablation resulted in reduced numbers of amplifying progenitors (type 2 a cells), in particular the numbers of proliferating $\left(\mathrm{PCNA}^{+}\right.$) amplifying progenitors. We hypothesize that the high percentage of Shh-responding cells in the amplifying progenitors (Ahn and Joyner, 2005), and/or the presence of other cilia-dependent signaling pathways, may account for the response to cilia deletion in this cell population.

Despite the fact that our data are consistent with the role of Shh acting as a mitogen for SGZ stem/progenitor cells, Shh might not be the only factor that maintains adult NSCs. For instance, recent work suggested that Notch/Hes5 signaling distinguishes two morphologically different hippocampal stem/progenitor cell populations (radial and nonradial) with differential response to various physiological stimuli (Lugert et al., 2010).

As a consequence of reduced proliferation in the SGZ, we observed a decrease in the number of newborn granule neurons in the DG. The reduction of proliferation of amplifying progenitors was associated with a decrease in newly born $\mathrm{DCX}^{+}$neurons (approximately twofold). Interestingly, a slightly greater reduction (approximately threefold) in the integration of these new granule neurons in the GCL (assessed 4 weeks after BrdU labeling) might indicate that primary cilia regulate survival of newborn granule cells in the DG. In addition, the cumulative ( 8 week) decrease in adult neurogenesis (approximately threefold) was found to be similar to the neurogenesis rate measured over $5 \mathrm{~d}$ of BrdU incorporation. Finally, the reduced levels of hippocampal neurogenesis observed in older animals (over 1 year) confirmed that reduction of neurogenesis remains constant over time.

Deletion of primary cilia in NSCs at embryonic stages causes a modest, but detectable reduction of proliferation in the SVZ (Han et al., 2008). In adults, stimulation or inhibition of Shh signaling pathway increased or decreased neural precursor proliferation in the SVZ, respectively (Machold et al., 2003; Palma et al., 2005). However, addition of exogenous Shh signaling modulators could affect other cell populations in the subventricular niche and, consequently, alter intercellular signaling in NSCs. In Ift20 mutants described in this study, primary cilia is ablated in adult $\mathrm{GFAP}^{+}$SVZ cells that are known to be proliferating NSCs (Kriegstein and Alvarez-Buylla, 2009) and exhibit a prominent pinwheel architecture of their apical primary cilia (Mirzadeh et al., 2008). In contrast to the reduced hippocampal neurogenesis, we only observed a modest reduction in cell proliferation at 12 months of age, suggesting that primary cilia do not contribute significantly to the maintenance of adult neurogenesis in the subventricular niche.

Adult hippocampal newborn neurons increase the efficiency of generating the new representations of spatial memories In humans, spatial memory disorders often underlie academic underachievement, difficulty with social relationships, and poor job performance (Cohen et al., 1993; Bloss et al., 2008). Recent evidence suggests that spatial memory processes modulate recruitment of newborn neurons into hippocampal networks (Trouche et al., 2009). Both behavioral (Kee et al., 2007) and computational (Aimone et al., 2009) studies have shown that newborn neurons are more likely than older neurons to be recruited into spatial memory networks. However, the consequences of integrating hippocampal newborn neurons to the 
spatial hippocampal network remain still elusive (Shors et al., 2002; Saxe et al., 2006; Garthe et al., 2009).

In the research presented here, we provide a novel approach that differs from previous ones based on inducible systems to acutely reduce adult neurogenesis (Zhang et al., 2008; Deng et al., 2009). Importantly, our behavioral studies examined the consequences of a constant reduction in adult hippocampal neurogenesis. Here, we evaluated a broad range of behavioral processes involving different complexities of spatial learning and memory, such as detection of subtle changes in the context, spatial resolution of a task, and efficiency of context and cued memory representations.

Extensive data emphasize the role of the hippocampus in processing information essential to recognition memory (Wan et al., 1999; Winters et al., 2004; Bachevalier and Nemanic, 2008) involving the relative familiarity of spatial distribution of items. Although recent data suggest that cells in the DG may also play a crucial role in spatial relationships comprising events (McHugh et al., 2007; Aimone et al., 2010), the precise contribution of adult hippocampal neurogenesis is widely debated (Saxe et al., 2006; Clelland et al., 2009). In our novel object location recognition paradigms, we tested mice for their ability to detect a change in the spatial patterning of objects relative to each other, but also for their ability to detect the displacement of a single object shifted to a close location, a more complex object exploration task that requires the mice to use distal spatial cues to discriminate similar contexts. In both cases, our results showed that Ift20 mutant mice had impaired spatial novelty recognition, suggesting that they had not developed a spatial representation of the objects during the familiarization trials.

Intriguingly, a delay in acquisition of spatial learning (Barnes maze) in animals with defective hippocampal neurogenesis was observed. Although Ift20 mutant mice eventually learned to complete the spatial task as wild-type mice did, they showed a specific difficulty in resolving the first trials, revealing a delay in acquisition of the spatial strategy. However, at the end of the experiment, Ift20 mutants performed the spatial task as well as wild-type mice, suggesting that they gathered enough spatial information to solve the task normally. Spatial memory retention test revealed similar long-term memory in Ift20 mutants and wild-type mice. Previous studies in which neurogenesis is abolished, however, have shown impairments in the long-term retention of spatial memory (Snyder et al., 2005; Imayoshi et al., 2008; Jessberger et al., 2009). A possible explanation for this discrepancy may lie in the different approach used; whereas previous approaches ablated adult hippocampal neurogenesis at a specific time point, our experimental strategy provided constantly reduced levels of neurogenesis, potentially modeling a different scenario in the levels of adult newborn neurons that integrate and form part of the pool of mature granule cells. In line with our long-term memory retention results, previous studies have reported the involvement of specific neocortical regions in the storage of information initially processed in the hippocampus (Wiltgen et al., 2004).

Hippocampal processing also plays a crucial role in the formation of contextual memory representations (Rudy et al., 2004). When the hippocampus is not functioning properly, these representations can be supported by feature representations (individual contextual stimuli) with the help of the cortex and amygdala (Rudy et al., 2004). Previous results in contextual learning with fear conditioning after ablation of adult neurogenesis were heterogeneous (Saxe et al., 2006; Dupret et al., 2008; Deng et al., 2009; Kitamura et al., 2009). In our model of constant reduced neurogenesis, contextual fear memory was slightly attenuated but failed to be abolished. We postulate that reduced neurogenesis may alter hippocampal circuitry, but other neural systems may be supporting contextual conditioning. Critically, Ift20 mutant mice showed altered hippocampus-independent cue conditioning. Enhanced conditioning response after the introduction of the shock-paired stimuli (tone plus light) in a different context suggests that Ift20 mutant mice are biased toward cue-based strategies. In fact, our results are consistent with the idea that, in the presence of impaired hippocampal function, contextual strategies are inoperable and force animals to use cuebased strategies (Nadel and Willner, 1980; Frankland et al., 1998).

Across all behavioral studies with our novel mouse model, we see that a coherent picture emerges. We speculate that constant reduction in the rate of integration of newborn neurons into hippocampal networks plays a critical role in efficiency of generating and subsequently distinguishing new spatial representations. If spatial representations are weakly processed in the hippocampal network, impaired discrimination of spatial novelty in a familiar context may occur. Thus, the generation of new spatial representations is insufficient to acquire an accurate spatial performance in short-term tests. However, when the spatial training is prolonged, the spatial representations can be successfully retrieved, producing a delay rather than a permanent deficiency in spatial learning. Finally, these deficits in contextual memory representations may be responsible for biasing animals toward cue-based strategies.

Ciliopathies are disorders characterized by the defects in structure or function of the primary cilia and display variable neurocognitive impairments (Singla and Reiter, 2006). Here, we described, for the first time, a genetic model of constant reduced adult neurogenesis due to the defects in primary cilia specifically ablated in the stem/progenitor cell compartment of the DG. Our findings highlight a novel aspect of primary cilia and adult neurogenesis and stimulate the search of new strategies for brain therapies to enhance neurogenesis in the context of aging and disease, with the aim of restoring cognitive function.

\section{Notes}

Supplemental material for this article is available at http://abs. sanfordburnham.org/centerandlabs/neuroagingstem/terskikhlab/Pages/ Home.aspx (Tersikh Laboratory URL at the Sanford-Burnham Medical Research Institute). This material has not been peer reviewed.

\section{References}

Ahn S, Joyner AL (2005) In vivo analysis of quiescent adult neural stem cells responding to Sonic hedgehog. Nature 437:894-897.

Aimone JB, Wiles J, Gage FH (2009) Computational influence of adult neurogenesis on memory encoding. Neuron 61:187-202.

Aimone JB, Deng W, Gage FH (2010) Adult neurogenesis: integrating theories and separating functions. Trends Cogn Sci 14:325-337.

Bach ME, Hawkins RD, Osman M, Kandel ER, Mayford M (1995) Impairment of spatial but not contextual memory in CaMKII mutant mice with a selective loss of hippocampal LTP in the range of the theta frequency. Cell 81:905-915.

Bachevalier J, Nemanic S (2008) Memory for spatial location and object-place associations are differently processed by the hippocampal formation, parahippocampal areas TH/TF and perirhinal cortex. Hippocampus 18:64-80.

Benice TS, Raber J (2008) Object recognition analysis in mice using nosepoint digital video tracking. J Neurosci Methods 168:422-430.

Biebl M, Cooper CM, Winkler J, Kuhn HG (2000) Analysis of neurogenesis and programmed cell death reveals a self-renewing capacity in the adult rat brain. Neurosci Lett 291:17-20.

Bloss CS, Delis DC, Salmon DP, Bondi MW (2008) Decreased cognition in children with risk factors for Alzheimer's disease. Biol Psychiatry 64:904-906.

Breunig JJ, Sarkisian MR, Arellano JI, Morozov YM, Ayoub AE, Sojitra S, Wang B, Flavell RA, Rakic P, Town T (2008) Primary cilia regulate hippocampal neurogenesis by mediating sonic hedgehog signaling. Proc Natl Acad Sci U S A 105:13127-13132.

Clelland CD, Choi M, Romberg C, Clemenson GD Jr, Fragniere A, Tyers P, Jessberger S, Saksida LM, Barker RA, Gage FH, Bussey TJ (2009) A func- 
tional role for adult hippocampal neurogenesis in spatial pattern separation. Science 325:210-213.

Cohen D, Eisdorfer C, Gorelick P, Paveza G, Luchins DJ, Freels S, Ashford JW, Semla T, Levy P, Hirschman R (1993) Psychopathology associated with Alzheimer's disease and related disorders. J Gerontol 48:M255-M260.

Corbit KC, Aanstad P, Singla V, Norman AR, Stainier DY, Reiter JF (2005) Vertebrate Smoothened functions at the primary cilium. Nature 437:1018-1021.

Crawley JN (1999) Behavioral phenotyping of transgenic and knockout mice: experimental design and evaluation of general health, sensory functions, motor abilities, and specific behavioral tests. Brain Res 835:18-26.

Deng W, Saxe MD, Gallina IS, Gage FH (2009) Adult-born hippocampal dentate granule cells undergoing maturation modulate learning and memory in the brain. J Neurosci 29:13532-13542.

Dupret D, Revest JM, Koehl M, Ichas F, De Giorgi F, Costet P, Abrous DN, Piazza PV (2008) Spatial relational memory requires hippocampal adult neurogenesis. PLoS One 3:e1959.

Encinas JM, Vaahtokari A, Enikolopov G (2006) Fluoxetine targets early progenitor cells in the adult brain. Proc Natl Acad Sci U S A 103:8233-8238.

Farioli-Vecchioli S, Saraulli D, Costanzi M, Pacioni S, Cinà I, Aceti M, Micheli L, Bacci A, Cestari V, Tirone F (2008) The timing of differentiation of adult hippocampal neurons is crucial for spatial memory. PLoS Biol 6:e246.

Follit JA, Tuft RA, Fogarty KE, Pazour GJ (2006) The intraflagellar transport protein IFT20 is associated with the Golgi complex and is required for cilia assembly. Mol Biol Cell 17:3781-3792.

Frankland PW, Cestari V, Filipkowski RK, McDonald RJ, Silva AJ (1998) The dorsal hippocampus is essential for context discrimination but not for contextual conditioning. Behav Neurosci 112:863-874.

Garcia AD, Doan NB, Imura T, Bush TG, Sofroniew MV (2004) GFAPexpressing progenitors are the principal source of constitutive neurogenesis in adult mouse forebrain. Nat Neurosci 7:1233-1241.

Garthe A, Behr J, Kempermann G (2009) Adult-generated hippocampal neurons allow the flexible use of spatially precise learning strategies. PLoS One 4:e5464.

Han YG, Spassky N, Romaguera-Ros M, Garcia-Verdugo JM, Aguilar A, Schneider-Maunoury S, Alvarez-Buylla A (2008) Hedgehog signaling and primary cilia are required for the formation of adult neural stem cells. Nat Neurosci 11:277-284.

Huangfu D, Liu A, Rakeman AS, Murcia NS, Niswander L, Anderson KV (2003) Hedgehog signalling in the mouse requires intraflagellar transport proteins. Nature 426:83-87.

Imayoshi I, Sakamoto M, Ohtsuka T, Takao K, Miyakawa T, Yamaguchi M, Mori K, Ikeda T, Itohara S, Kageyama R (2008) Roles of continuous neurogenesis in the structural and functional integrity of the adult forebrain. Nat Neurosci 11:1153-1161.

Jessberger S, Clark RE, Broadbent NJ, Clemenson GD Jr, Consiglio A, Lie DC, Squire LR, Gage FH (2009) Dentate gyrus-specific knockdown of adult neurogenesis impairs spatial and object recognition memory in adult rats. Learn Mem 16:147-154.

Jonassen JA, San Agustin J, Follit JA, Pazour GJ (2008) Deletion of IFT20 in the mouse kidney causes misorientation of the mitotic spindle and cystic kidney disease. J Cell Biol 183:377-384.

Kee N, Teixeira CM, Wang AH, Frankland PW (2007) Preferential incorporation of adult-generated granule cells into spatial memory networks in the dentate gyrus. Nat Neurosci 10:355-362.

Kitamura T, Saitoh Y, Takashima N, Murayama A, Niibori Y, Ageta H, Sekiguchi M, Sugiyama H, Inokuchi K (2009) Adult neurogenesis modulates the hippocampus-dependent period of associative fear memory. Cell 139:814-827.

Kriegstein A, Alvarez-Buylla A (2009) The glial nature of embryonic and adult neural stem cells. Annu Rev Neurosci 32:149-184.

Lai K, Kaspar BK, Gage FH, Schaffer DV (2003) Sonic hedgehog regulates adult neural progenitor proliferation in vitro and in vivo. Nat Neurosci 6:21-27.

Lugert S, Basak O, Knuckles P, Haussler U, Fabel K, Götz M, Haas CA, Kempermann G, Taylor V, Giachino C (2010) Quiescent and active hippocampal neural stem cells with distinct morphologies respond selectively to physiological and pathological stimuli and aging. Cell Stem Cell 6:445-456.

Machold R, Hayashi S, Rutlin M, Muzumdar MD, Nery S, Corbin JG, GritliLinde A, Dellovade T, Porter JA, Rubin LL, Dudek H, McMahon AP,
Fishell G (2003) Sonic hedgehog is required for progenitor cell maintenance in telencephalic stem cell niches. Neuron 39:937-950.

McHugh TJ, Jones MW, Quinn JJ, Balthasar N, Coppari R, Elmquist JK, Lowell BB, Fanselow MS, Wilson MA, Tonegawa S (2007) Dentate gyrus NMDA receptors mediate rapid pattern separation in the hippocampal network. Science 317:94-99.

Mignone JL, Kukekov V, Chiang AS, Steindler D, Enikolopov G (2004) Neural stem and progenitor cells in nestin-GFP transgenic mice. J Comp Neurol 469:311-324.

Mirzadeh Z, Merkle FT, Soriano-Navarro M, Garcia-Verdugo JM, AlvarezBuylla A (2008) Neural stem cells confer unique pinwheel architecture to the ventricular surface in neurogenic regions of the adult brain. Cell Stem Cell 3:265-278.

Nadel L, Willner J (1980) Context and conditioning: a place for space. Physiol Psychol 8:218-228.

Novak A, Guo C, Yang W, Nagy A, Lobe CG (2000) Z/EG, a double reporter mouse line that expresses enhanced green fluorescent protein upon Cremediated excision. Genesis 28:147-155.

Palma V, Lim DA, Dahmane N, Sánchez P, Brionne TC, Herzberg CD, Gitton Y, Carleton A, Alvarez-Buylla A, Ruiz i Altaba A (2005) Sonic hedgehog controls stem cell behavior in the postnatal and adult brain. Development 132:335-344.

Porsolt RD, Le Pichon M, Jalfre M (1977) Depression: a new animal model sensitive to antidepressant treatments. Nature 266:730-732.

Rohatgi R, Milenkovic L, Scott MP (2007) Patched1 regulates hedgehog signaling at the primary cilium. Science 317:372-376.

Rudy JW, Huff NC, Matus-Amat P (2004) Understanding contextual fear conditioning: insights from a two-process model. Neurosci Biobehav Rev 28:675-685.

Saxe MD, Battaglia F, Wang JW, Malleret G, David DJ, Monckton JE, Garcia AD, Sofroniew MV, Kandel ER, Santarelli L, Hen R, Drew MR (2006) Ablation of hippocampal neurogenesis impairs contextual fear conditioning and synaptic plasticity in the dentate gyrus. Proc Natl Acad Sci U S A 103:17501-17506.

Seri B, García-Verdugo JM, McEwen BS, Alvarez-Buylla A (2001) Astrocytes give rise to new neurons in the adult mammalian hippocampus. J Neurosci 21:7153-7160.

Seri B, García-Verdugo JM, Collado-Morente L, McEwen BS, Alvarez-Buylla A (2004) Cell types, lineage, and architecture of the germinal zone in the adult dentate gyrus. J Comp Neurol 478:359-378.

Shors TJ, Townsend DA, Zhao M, Kozorovitskiy Y, Gould E (2002) Neurogenesis may relate to some but not all types of hippocampal-dependent learning. Hippocampus 12:578-584.

Singla V, Reiter JF (2006) The primary cilium as the cell's antenna: signaling at a sensory organelle. Science 313:629-633.

Snyder JS, Hong NS, McDonald RJ, Wojtowicz JM (2005) A role for adult neurogenesis in spatial long-term memory. Neuroscience 130:843-852.

Steiner B, Klempin F, Wang L, Kott M, Kettenmann H, Kempermann G (2006) Type-2 cells as link between glial and neuronal lineage in adult hippocampal neurogenesis. Glia 54:805-814.

Suh H, Consiglio A, Ray J, Sawai T, D’Amour KA, Gage FH (2007) In vivo fate analysis reveals the multipotent and self-renewal capacities of Sox $2+$ neural stem cells in the adult hippocampus. Cell Stem Cell 1:515-528.

Trouche S, Bontempi B, Roullet P, Rampon C (2009) Recruitment of adultgenerated neurons into functional hippocampal networks contributes to updating and strengthening of spatial memory. Proc Natl Acad Sci U S A 106:5919-5924.

van Praag H, Schinder AF, Christie BR, Toni N, Palmer TD, Gage FH (2002) Functional neurogenesis in the adult hippocampus. Nature 415:1030-1034.

Wan H, Aggleton JP, Brown MW (1999) Different contributions of the hippocampus and perirhinal cortex to recognition memory. J Neurosci 19:1142-1148.

Wiltgen BJ, Brown RA, Talton LE, Silva AJ (2004) New circuits for old memories: the role of the neocortex in consolidation. Neuron 44:101-108.

Winters BD, Forwood SE, Cowell RA, Saksida LM, Bussey TJ (2004) Double dissociation between the effects of peri-postrhinal cortex and hippocampal lesions on tests of object recognition and spatial memory: heterogeneity of function within the temporal lobe. J Neurosci 24:5901-5908.

Zhang CL, Zou Y, He W, Gage FH, Evans RM (2008) A role for adult TLXpositive neural stem cells in learning and behaviour. Nature 451:1004-1007. 